\title{
Optimization of Two-Dimensional Homonuclear Relayed Coherence Transfer NMR Spectroscopy
}

\author{
AD BAX* AND GARY DROBNY $\dagger$ \\ *Laboratory of Chemical Physics, National Institute of Arthritis, Diabetes and Digestive \\ and Kidney Diseases, National Institutes of Health, Bethesda, Maryland 20205, and \\ †Department of Chemistry, University of Washington, Seattle, Washington 98195
}

Received August 1, 1984

\begin{abstract}
The optimization of the recently introduced two-dimensional homonuclear RELAY experiment is described. Practical guidelines for optimization of experimental parameters and data processing are presented. The effectiveness of relayed magnetization transfer is found to be strongly dependent on the type of spin system. Explicit calculations predict the relative intensities of RELAY cross peaks for a number of commonly occurring systems. The feasibility of doubly relayed magnetization transfer experiments is discussed and demonstrated for a sample of gramacidin-S and for the trinucleotide A2'-5'A2'-5'A. (c) 1985 Academic Press, Inc.
\end{abstract}

\section{INTRODUCTION}

The homonuclear RELAY experiment was first introduced by Eich, Bodenhausen, and Ernst (1). The experiment is basically an extension of the more familiar homonuclear shift correlation experiment $(2-10)$, often referred to as the COSY experiment. In the COSY experiment, magnetization transfer is limited to pairs of protons that have a mutual scalar interaction. The homonuclear RELAY experiment allows transfer between protons that are not mutually coupled, provided that both are coupled to a third "central" proton. It has been demonstrated that the experiment has great promise for the study of peptides and small proteins $(11,12)$. However, the RELAY experiment is inherently less sensitive than the COSY experiment, lineshapes in the 2D RELAY spectrum are more complicated, and intensities of peaks are generally poorly understood. We present a detailed analysis of the mechanism of the RELAY experiment, and will discuss the efficiency of the experiment for a number of different spin systems. General guidelines for the optimization of the experiment will be presented.

In our analysis of the RELAY experiment we use the recently introduced operator formalism approach (13), because this provides physical insight into the mechanism of the experiment and avoids the use of the more cumbersome density matrix approach. To agree with the nomenclature used by Sorensen et al. (13), we define a $90_{x}^{\circ}$ pulse as a pulse that rotates magnetization from the positive $z$ axis to the negative $y$ axis. 


\section{THE HOMONUCLEAR RELAY EXPERIMENT}

The basic pulse scheme of the homonuclear RELAY experiment is set out in Fig. 1. The effect of this sequence will be explained below, for the simplest case of a weakly scalar coupled AMX proton spin system with $J_{\mathrm{AX}}=0$. First the transfer of magnetization during the mixing period from $A$ to $X$ will be considered, as this is the process that provides the crucial information in a RELAY spectrum. A sequence with all $90^{\circ} \mathrm{rf}$ pulses applied along the $x$ axis of the rotating frame and the $180^{\circ}$ pulse applied along the $y$ axis will be considered. For reasons of simplicity, effects of transverse relaxation will be omitted unless explicitly stated.

The first $90_{x}^{\circ}$ pulse generates transverse A-spin magnetization along the $-y$ axis $\left(-I_{A y}\right)$.This magnetization then evolves during the evolution period under the Hamiltonian $\Omega_{\mathrm{A}} I_{z}+2 \pi J_{\mathrm{AM}} I_{\mathrm{A} z} I_{\mathrm{M} z}$ :

$$
\begin{aligned}
I_{\mathrm{A} y} \stackrel{\Omega_{\mathrm{A} 1} I_{\mathrm{A} z}+2 \pi J_{\mathrm{AM}} I_{\mathrm{A} z} I_{\mathrm{M} z} t_{1}}{\longrightarrow} & {\left[-I_{\mathrm{A} y} \cos \left(\Omega_{\mathrm{A}} t_{1}\right)+I_{\mathrm{A} x} \sin \left(\Omega_{\mathrm{A}} t_{1}\right)\right] \cos \left(\pi J_{\mathrm{AM}} t_{1}\right) } \\
& +2\left[I_{\mathrm{A} x} \cos \left(\Omega_{\mathrm{A}} t_{1}\right)+I_{\mathrm{A} y} \sin \left(\Omega_{\mathrm{A}} t_{1}\right)\right] I_{\mathrm{Mz}} \sin \left(\pi J_{\mathrm{AM}} t_{1}\right) .
\end{aligned}
$$

The first term at the right-hand side of the arrow denotes the in-phase A-spin magnetization. The second term is the term of interest, since it represents the antiphase A-spin magnetization that can be transferred to proton $M$ by a $90^{\circ}$ pulse. The second $90^{\circ}$ pulse, applied at the end of the evolution period, transfers the antiphase A-spin magnetization according to

$$
I_{\mathrm{M} z}\left[I_{\mathrm{A} x} \cos \left(\Omega_{\mathrm{A}} t_{1}\right)+I_{\mathrm{A} y} \sin \left(\Omega_{\mathrm{A}} t_{1}\right)\right] \stackrel{\pi / 2\left(I_{\mathrm{A} x}+I_{\mathrm{M} x}\right)}{\longrightarrow}-I_{\mathrm{M} y}\left[I_{\mathrm{A} x} \cos \left(\Omega_{\mathrm{A}} t_{1}\right)+I_{\mathrm{A} z} \sin \left(\Omega_{\mathrm{A}} t_{1}\right)\right] .
$$

The first term at the right-hand side of the arrow contains the product $I_{\mathrm{Ax}} I_{\mathrm{M} y}$, and therefore denotes two-spin (zero- and double-quantum) coherence. As will be discussed later, this term cannot contribute to the transfer of magnetization from $A$ to $\mathrm{X}$. The second term denotes the $\mathrm{M}$-spin magnetization that has been transferred from $A$, which is in antiphase with respect to spin $A$, and in phase with respect to spin $\mathrm{X}$, along the $\pm y$ axis. The $180^{\circ}$ pulse at the midpoint of the evolution period removes all chemical shift effects, and the second term in expression [2] evolves according to

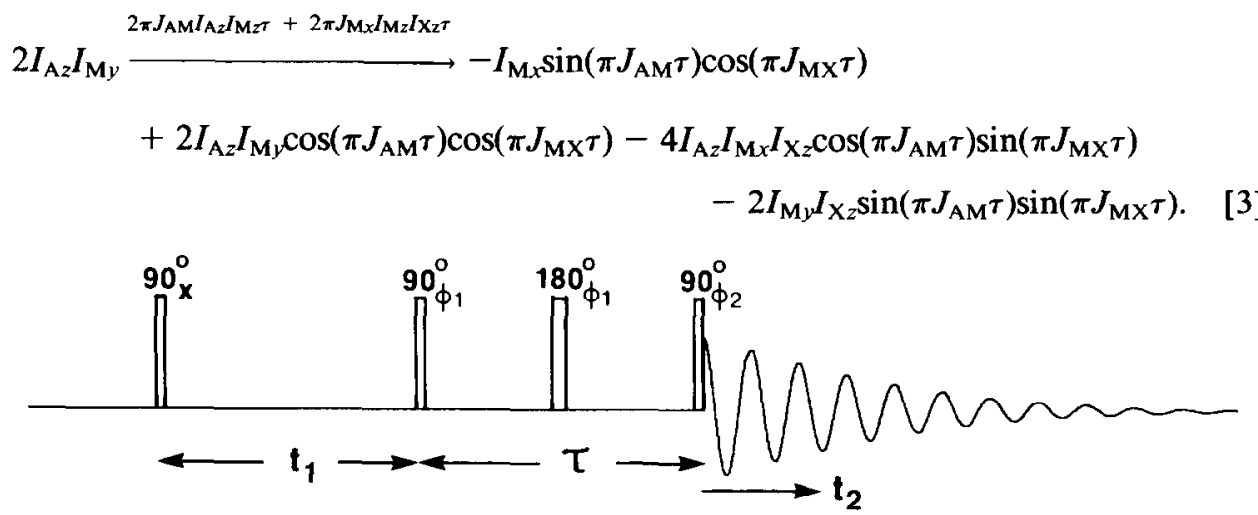

FIG. 1. Pulse scheme of the homonuclear RELAY experiment. The phases of the if pulses and of the receiver are cycled according to Table 1 . 
The terms at the right-hand side of the arrow in [3] denote the M-spin magnetization at the end of the mixing period, just before the final $90^{\circ}$ mixing pulse. Although, as will be shown below, only the fourth term contributes to the AX cross multiplet in the $2 \mathrm{D}$ spectrum, the fate of the other three terms will be discussed first. For convenience, the sine and cosine coefficients will be omitted. The first term, $I_{M x}$ denotes in-phase $\mathrm{X}$-spin magnetization along the positive $x$ axis. The final $90_{x}^{\circ}$ pulse will not affect this magnetization, and this in-phase $M$ spin magnetization will contribute to an AM cross peak in the $2 \mathrm{D}$ spectrum with in-phase multiplet components in the $F_{2}$ dimension and antiphase in the $F_{1}$ dimension. The second term, $I_{\mathrm{My}} I_{\mathrm{A} z}$, is converted into $-I_{\mathrm{Mz}} I_{\mathrm{A} y}$ by the final $90^{\circ}{ }_{x}$ pulse, and represents transfer from $\mathrm{A}$ to $\mathrm{M}$ to $\mathrm{A}$, and therefore contributes to the diagonal peak in the $2 \mathrm{D}$ spectrum. The third term, $I_{\mathrm{M} x} I_{\mathrm{A} z} I_{\mathrm{X} z}$ is converted into three-spin coherence, $I_{\mathrm{M} x} I_{\mathrm{A} y} I_{\mathrm{X} y}$, which is not observable during the detection time, $t_{2}$, and therefore does not contribute to the $2 \mathrm{D}$ spectrum.

It is the fourth term in expression [3], $I_{\mathrm{M} y} I_{\mathrm{X}_{z}}$, that is converted into transverse $\mathrm{X}$-spin magnetization that is in antiphase with respect to spin $\mathrm{M},-I_{\mathrm{Mz}} I_{\mathrm{X} y}$. The complete expression for this relayed signal, with quadrature detection during $t_{2}$, is given by

$$
S_{\mathrm{AX}}\left(t_{1}, t_{2}\right)=\sin \left(\pi J_{\mathrm{AM}} \tau\right) \sin \left(\pi J_{\mathrm{MX}} \tau\right) \sin \left(\pi J_{\mathrm{AM}} t_{1}\right) \sin \left(\pi J_{\mathrm{MX}} t_{2}\right) \sin \left(\Omega_{\mathrm{A}} t_{1}\right) \exp \left(i \Omega_{\mathrm{X}} t_{2}\right)
$$

In full analogy to phase cycling in the more familiar COSY experiment (4-10), incrementing the phases of all pulses in the mixing period by $90^{\circ}$ (i.e., $90^{\circ}{ }_{y} \tau / 2-$ $\left.180^{\circ}{ }_{-x}-\tau / 2-90^{\circ}{ }_{y}\right)$, gives a $S_{\mathrm{AX}}\left(t_{1}, t_{2}\right)$ signal of the form

$$
S_{\mathrm{AX}}\left(t_{1}, t_{2}\right)=-i \sin \left(\pi J_{\mathrm{AM}} \tau\right) \sin \left(\pi J_{\mathrm{MX}} \tau\right) \sin \left(\pi J_{\mathrm{AM}} t_{1}\right) \sin \left(\pi J_{\mathrm{MX}} t_{2}\right) \cos \left(\Omega_{\mathrm{A}} t_{1}\right) \exp \left(i \Omega_{\mathrm{X}} t_{2}\right) .
$$

The difference of Eqs. [4] and [5], rearranging of the order of the various terms, gives

$$
S_{\mathrm{AX}}\left(t_{1}, t_{2}\right)=-i \sin \left(\pi J_{\mathrm{AM}} \tau\right) \sin \left(\pi J_{\mathrm{MX}} \tau\right) \sin \left(\pi J_{\mathrm{AM}} t_{1}\right) \sin \left(\pi J_{\mathrm{MX}} t_{2}\right) \exp \left(-i \Omega_{\mathrm{A}} t_{1}\right) \exp \left(i \Omega_{\mathrm{X}} t_{2}\right)
$$

Hence, phase modulation is easily feasible, and is completely analogous with phase cycling commonly used in the COSY experiment (4-10). An 8-step cycle is used in practice (Table 1) to suppress possible artifacts, and, as will be explained later, to decrease the size of the diagonal multiplets. As is commonly done in many $2 \mathrm{D}$ experiments, the entire 8-step cycle can be repeated four times, with phases of all rf pulses and receiver incremented by $90^{\circ}$ each time, to further suppress possible artifacts (14). Cycling of the phase of the $180^{\circ}$ pulse can remove part of its " $90^{\circ}$ character" (15), but will generally not be necessary in this experiment, since the main effect of a small error in the $180^{\circ}$ pulse is to cause small amplitude distortions, and no spurious resonances (apart from possibly doubly relayed very weak resonances). 
TABLE 1

The Phases of the If Pulses and of the Receiver in the Various Steps of the Homonuclear RELAY Experiment, Sketched in Fig. $1^{a}$

\begin{tabular}{lrrc}
\hline Step & $\phi_{1}$ & $\phi_{2}$ & Receiver \\
\hline 1 & $x$ & $x$ & + \\
2 & $x$ & $-x$ & + \\
3 & $y$ & $y$ & - \\
4 & $y$ & $-y$ & - \\
5 & $-x$ & $-x$ & + \\
6 & $-x$ & $x$ & + \\
7 & $-y$ & $-y$ & - \\
8 & $-y$ & $y$ & - \\
\hline
\end{tabular}

\footnotetext{
a The entire 8-step experiment should be repeated four times, with the phases of all pulses and of the receiver incremented by $90^{\circ}$ each time.
}

\section{ORIGIN OF OTHER PEAKS IN THE 2D SPECTRUM}

First, we will explain why AM two-spin coherence, created by the second $90^{\circ}$ pulse, cannot result in an AX relay peak. This two-spin coherence is of the form $I_{\mathrm{A} \alpha} I_{\mathrm{M} \beta}$ where $\alpha$ and $\beta$ can be either $x$ or $y$. This coherence becomes in antiphase with respect to spin $\mathrm{X}$ to give terms of the form $I_{\mathrm{A \alpha}} I_{\mathrm{M} \beta} I_{\mathrm{Xz}} \sin \left(\pi J_{\mathrm{MX}} \tau\right)$. The third mixing pulse can then only create transverse $\mathrm{X}$-spin magnetization that is of the form $I_{\mathrm{A} z} I_{\mathrm{M} z} I_{\mathrm{X} y}$. Because $\mathrm{A}$ and $\mathrm{X}$ are not coupled, the $I_{\mathrm{A} z}$ term in this product prevents this "magnetization" from being observable.

There are four different pathways (16) that can contribute to diagonal multiplets, schematically represented for evolution, mixing, and detection periods as

$$
\begin{aligned}
& A_{t r} \rightarrow A_{z} \rightarrow A_{t r} \\
& A_{t r} \rightarrow A_{t r} \rightarrow A_{t r} \\
& A_{t r} \rightarrow A M \rightarrow A_{t r} \\
& A_{t r} \rightarrow M_{t r} \rightarrow A_{t r}
\end{aligned}
$$

where $A_{t r}$ and $M_{t r}$ denote transverse $A$ and $M$ spin magnetization, $A_{z}$ is longitudinal $A$ spin magnetization and AM is two-spin AM coherence. The first and third of those four pathways ([7a] and [7c]), are eliminated by alternation of the phase of the final $90^{\circ}$ pulse along the $\pm x$ axis (Table 1). The sum of the remaining two pathways is given by

$$
\begin{aligned}
S_{\mathrm{AA}}\left(t_{1}, t_{2}\right) & =-\cos \left(\pi J_{\mathrm{AM}} \tau\right) \cos \left(\pi J_{\mathrm{AM}} t_{1}\right) \cos \left(\pi J_{\mathrm{AM}} t_{2}\right) \exp \left(-i \Omega_{\mathrm{A}} t_{1}\right) \exp \left(i \Omega_{\mathrm{A}} t_{2}\right) \\
& +\cos \left(\pi J_{\mathrm{AM}} \tau\right) \cos \left(\pi J_{\mathrm{MX}} \tau\right) \sin \left(\pi J_{\mathrm{AM}} t_{1}\right) \sin \left(\pi J_{\mathrm{AM}} t_{2}\right) \exp \left(-i \Omega_{\mathrm{A}} t_{1}\right) \exp \left(i \Omega_{\mathrm{A}} t_{2}\right)
\end{aligned}
$$

It is seen from this expression, that the diagonal multiplet consists of two contributions with different phases, and it will therefore generally be impossible to phase this multiplet to the pure absorption mode. 
The "COSY multiplet" in the 2D RELAY spectrum occurs via two different pathways:

The pathway

$$
\begin{aligned}
& A_{t r} \rightarrow A_{t r} \rightarrow M_{t r} \\
& A_{t r} \rightarrow M_{t r} \rightarrow M_{t r} .
\end{aligned}
$$

$$
\mathrm{A}_{\mathrm{tr}} \rightarrow \mathrm{AM} \rightarrow \mathrm{M}_{\mathrm{tr}}
$$

does not give a contribution in the case of phase cycling according to Table 1, assuming perfect if pulses and phase shifts.

The magnetization transferred from $A$ to $M$ is then described by

$$
\begin{aligned}
& S_{\mathrm{AM}}\left(t_{1}, t_{2}\right)=-\sin \left(\pi J_{\mathrm{AM}} \tau\right) \cos \left(\pi J_{\mathrm{AM}} t_{1}\right) \sin \left(\pi J_{\mathrm{AM}} t_{2}\right) \cos \left(\pi J_{\mathrm{MX}} t_{2}\right) \exp \left(-i \Omega_{\mathrm{A}} t_{1}\right) \exp \left(i \Omega_{\mathrm{M}} t_{2}\right) \\
& \quad+\sin \left(\pi J_{\mathrm{AM}} \tau\right) \cos \left(\pi J_{\mathrm{MX}} \tau\right) \sin \left(\pi J_{\mathrm{AM}} t_{1}\right) \cos \left(\pi J_{\mathrm{AM}} t_{2}\right) \cos \left(\pi J_{\mathrm{MX}} t_{2}\right) \exp \left(-i \Omega_{\mathrm{A}} t_{1}\right) \exp \left(i \Omega_{\mathrm{M}} t_{2}\right)
\end{aligned}
$$

This signal represents the superposition of an "in-phase $F_{1}$, antiphase $F_{2}$ " and an "antiphase $F_{1}$, in-phase $F_{2}$ " multiplet which are $90^{\circ}$ out of phase relative to each other, and can in general not be phased to the $2 \mathrm{D}$ absorption mode.

\section{OPTIMIZATION FOR AN AMX SPIN SYSTEM}

In optimizing the homonuclear RELAY experiment, the aspects to consider are (a) optimizing intensity of relayed magnetization, (b) minimizing intensity of COSY and diagonal peaks, and (c) optimizing resolution in the $2 \mathrm{D}$ spectrum.

(a) The intensity, $I$, of the relayed magnetization follows directly from expression [4], and has the proportionality

$$
I \sim \sin \left(\pi J_{\mathrm{AM}} \tau\right) \sin \left(\pi J_{\mathrm{MX}} \tau\right) \exp \left(-\tau / T_{2}\right)
$$

where $T_{2}$ is the transverse relaxation time of spin $\mathrm{M}$. For the case where $J_{\mathrm{AM}}$ and $J_{\mathrm{MX}}$ differ by less than a factor of two in magnitude, and $J_{\mathrm{AM}}, J_{\mathrm{MX}} \gg T_{2}^{-1}$, the optimum $\tau$ value is near the condition

$$
\tau=\left(J_{\mathrm{AM}}+J_{\mathrm{MX}}\right)^{-1} .
$$

In the case where $J_{\mathrm{AM}}, J_{\mathrm{MX}} \ll T_{2}^{-1}$, the optimum $\tau$ value is near the condition

$$
\tau=2 T_{2} .
$$

In the important intermediate case where $J$ is of the order of $T_{2}^{-1}$, a value slightly (10-30\%) shorter than given in Eq. [11b] will optimize the amount of relayed magnetization. Figure 2 shows a graphical presentation of the relayed intensity as a function of mixing time, for various values of $T_{2}$. From Eq. [6] it is seen that the amount of relayed magnetization is proportional to $\sin \left(\pi J_{\mathrm{AM}} t_{1}\right) \sin \left(\pi J_{\mathrm{Mx}} t_{2}\right)$. For acquisition times in the $t_{1}$ and $t_{2}$ dimension ( $t_{1 \max }$ and $t_{2 \max }$, respectively) on the order of $1 /\left(J_{\mathrm{HH}}\right)$, optimum "matched" filtering $(17)$ is therefore a nonshifted sinebell function (18), possibly in combination with exponential or Gaussian weighting to take relaxation effects into account. The similarly shaped convolution difference 


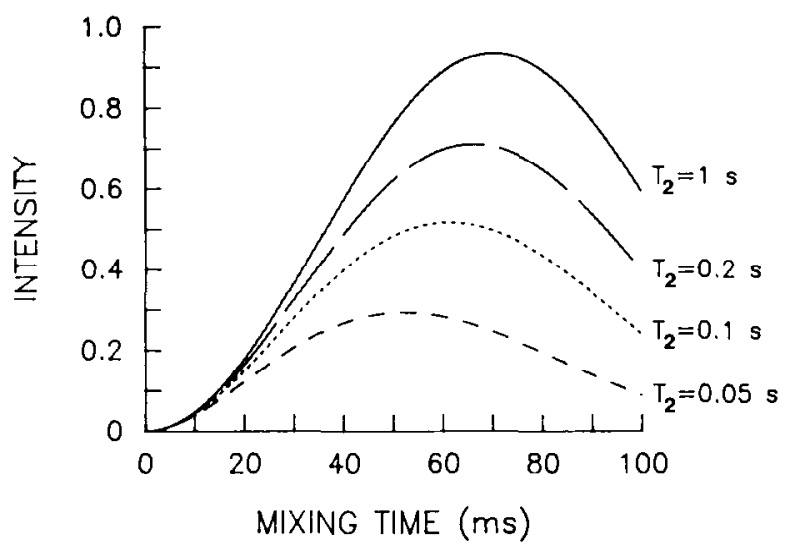

FiG. 2. Intensity of the AX RELAY cross multiplet in an AMX system as a function of the mixing time for various values of the transverse relaxation time, $T_{2}$. Intensity " 1 " corresponds approximately to the intensity that would be observed for an $\mathrm{A}^{\prime} \mathrm{X}^{\prime}$ system with $J_{\mathrm{AX}}=\left(\left|J_{\mathrm{AM}}\right|+\left|J_{\mathrm{MX}}\right|\right) / 2$ in a regular COSY experiment with identical acquisition and data processing parameters. $J_{\mathrm{AM}}=J_{\mathrm{MX}}=7.0 \mathrm{~Hz}$.

filtering function (19) and pseudo-echo window (20) can also be used for this purpose, but are often harder to optimize than is the use of a nonshifted sine-bell function.

(b) The intensity of diagonal and COSY peaks will be close to their minimum for a $\tau$ value given in Eq. [11b]. Parts of the time domain signals of diagonal peaks are in phase for $t_{1}=0$ and for $t_{2}=0$ (Eq. [8] and [10]), this means, they have a $\cos \left(\pi J_{\mathrm{AM}} t_{1}\right)$ or $\cos \left(\pi J_{\mathrm{AM}} t_{2}\right)$ dependence. Those parts will be attenuated by the use of the sine bell filtering function.

(c) Resolution in the absolute-value mode presentation is close to optimum if a sine-bell filtering function in both dimensions is used prior to Fourier transformation. Long acquisition times in the $t_{1}$ and $t_{2}$ dimensions in principle provide high resolution, but for lengths larger than $1 / J_{\mathrm{HH}}$, this does usually not significantly enhance the intensity of a cross multiplet anymore but merely resolves individual components in the cross multiplet. This higher resolution can be useful when the method is applied to molecules with severe overlap of cross multiplets but generally is not recommended for the study of large molecules which often have short $T_{2}$ values, because of the dramatic effect on sensitivity. Therefore, in general, values for $t_{1 \max }$ and $t_{2 \max }$ of the order of 100 to $150 \mathrm{~ms}$ provide optimum results, and require only a modest data matrix size.

\section{OTHER SPIN SYSTEMS}

The discussions given above were restricted to the simple case of an AMX spin system. In practice, however, often more complicated spin systems are encountered. Optimization of the choice for the $\tau$ delay will be discussed for a number of commonly occurring spin systems. The simple case of an AMX subsystem, discussed above, is also applicable to systems where proton $\mathrm{A}$ and/or $\mathrm{X}$ are coupled to protons other than $M$. The only restriction on the AMX case is that proton $M$ is only coupled to two protons, $\mathrm{A}$ and $\mathrm{X}$. 
$A M X_{2}$ spin system. The $\mathrm{AMX}_{2}$ system sometimes applies to cases where one transfers from the amide proton to the two $\beta$ protons in a number of amino acids, provided that the two $\beta$ protons are magnetically equivalent. Using the operator formalism approach (14), a relayed transfer efficiency function $F(\tau)$ can be derived, given by

$$
F(\tau)=\sin \left(\pi J_{\mathrm{AM}} \tau\right) \sin \left(2 \pi J_{\mathrm{MX}} \tau\right)
$$

For the case where $J_{\mathrm{AM}}=J_{\mathrm{MX}}$, this function is sketched in Fig. 3a.

$A M X_{3}$ spin system. This system applies, for example, for transfer from an amide proton to the methyl group in alanine residues. The transfer function is given by

$$
F(\tau)=3 \sin \left(\pi J_{\mathrm{AM}} \tau\right)\left[\sin \left(\pi J_{\mathrm{MX}} \tau\right)+\sin \left(3 \pi J_{\mathrm{MX}} \tau\right)\right] / 4
$$

This function is sketched in Fig. $3 \mathrm{~b}$.

$A_{3} M Q X_{3}$ spin system. This system is appropriate for the magnetization transfer between the two inequivalent methyl groups in valine residues. The transfer efficiency is in this case described by

$$
F(\tau)=9\left[\sin \left(\pi J_{\mathrm{AM}} \tau\right)+\sin \left(3 \pi J_{\mathrm{AM}} \tau\right)\right]\left[\sin \left(\pi J_{\mathrm{MX}} \tau\right)+\sin \left(3 \pi J_{\mathrm{MX}} \tau\right)\right] \cos \left(\pi J_{\mathrm{MQ}} \tau\right) / 16
$$

This function is sketched in Fig. 3c. Note that very intense RELAY peaks can be observed for $\tau$ values on the order of $25 \mathrm{~ms}$, whereas the intensity rapidly drops to zero for larger values.

$A M Q_{3} X_{3}$ spin system. For the case where the chemical shifts of the two methyl groups of a valine residue differ by more than several hertz, the spin system must be considered as an $\mathrm{AMQ}_{3} \mathrm{X}_{3}$ system. In this case the transfer efficiency is given by

$$
F(\tau)=3 \sin \left(\pi J_{\mathrm{AM}} \tau\right)\left[\sin \left(\pi J_{\mathrm{MX}} \tau\right)+\sin \left(3 \pi J_{\mathrm{MX}} \tau\right)\right]\left[3 \cos \left(\pi J_{\mathrm{MZ}} \tau\right)+\cos \left(3 \pi J_{\mathrm{MZ}} \tau\right)\right] / 16
$$

This function is sketched in Fig. 3d.

AMQX spin system. The AMQX spin system occurs very commonly. Two different cases can be distinguished:

(1) Both spins $A$ and $X$ are coupled to both spins $M$ and $Q$. This situation occurs, for example, in leucine, for transfer from the $\alpha$ to the $\gamma$ proton, provided that the two $\beta$ protons are nonequivalent and weakly coupled. This type of spin system also applies to deoxynucleotides, for transfer from the $1^{\prime}$ to the $3^{\prime}$ proton. The transfer function in this case is the sum of two contributions, transfer via $M$, and transfer via $\mathrm{Q}$ :

$$
F(\tau)=\sin \left(\pi J_{\mathrm{AM}} \tau\right) \sin \left(\pi J_{\mathrm{MX}} \tau\right) \cos \left(\pi J_{\mathrm{MQ}} \tau\right)+\sin \left(\pi J_{\mathrm{AQ}} \tau\right) \sin \left(\pi J_{\mathrm{QX}} \tau\right) \cos \left(\pi J_{\mathrm{MQ}} \tau\right)
$$

The geminal coupling $J_{\mathrm{MQ}}$ is usually on the order of $12-16 \mathrm{~Hz}$ and signal nulling of the relay peaks will occur for $\tau$ values on the order of $35 \mathrm{~ms}$. The transfer efficiency is sketched in Fig. 3e. Equation [16] describes the total magnetization transfer from $A$ to $X$. Even though this function has a rather large value for a relatively long duration of the mixing time, in practice the RELAY cross peaks usually have low intensity. This is due to the fact that the multiplet structure of $A$ and $M$, over which the relayed magnetization is distributed, are split by both $M$ and $Q$, halving their intensities. 

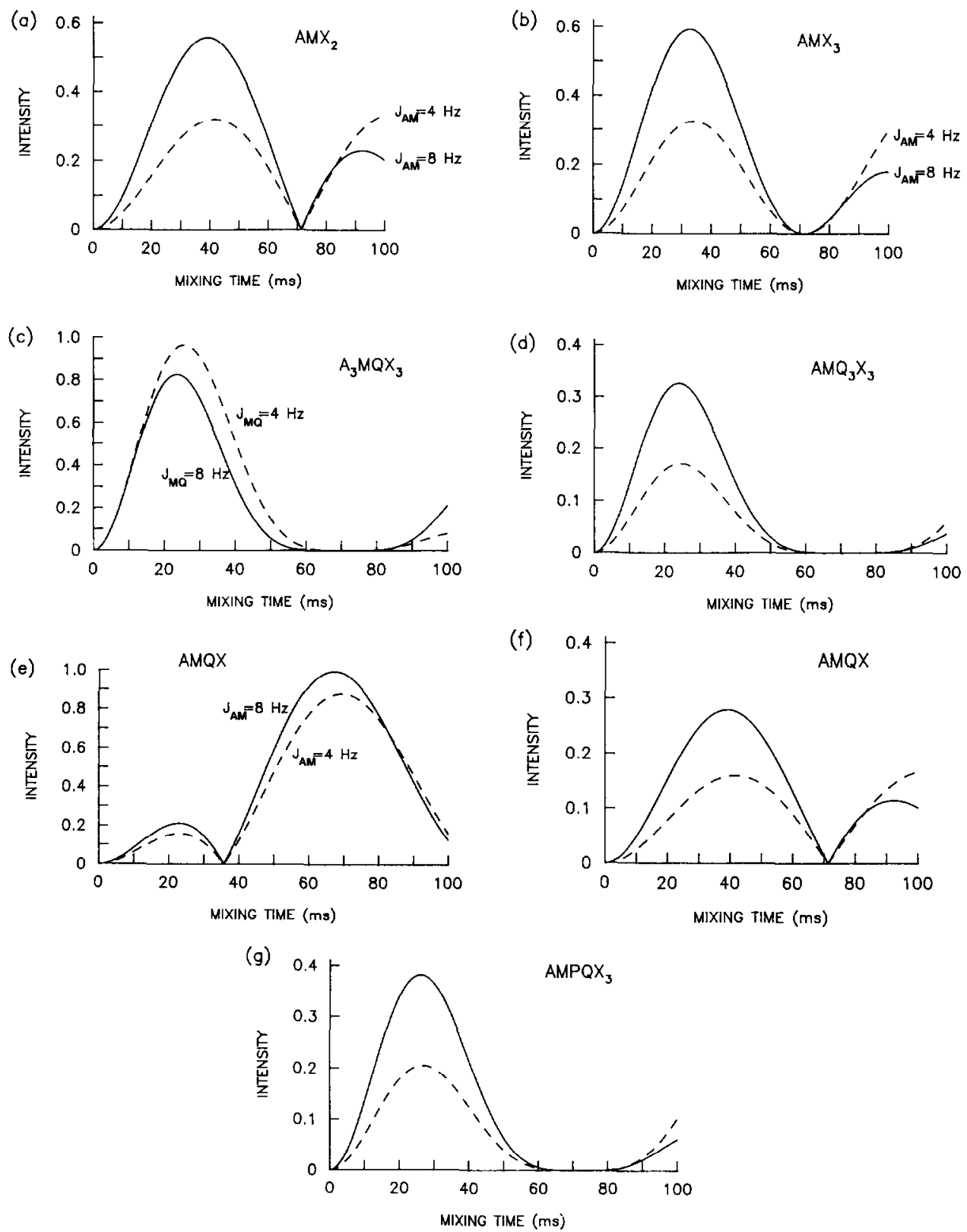

FIG. 3. Intensity of the AX RELAY cross multiplet as a function of mixing time for various spin systems in which $J_{\mathrm{AX}}=0$, and $T_{2}=100 \mathrm{~ms}$. (a) $\mathrm{AMX}_{2}$ system with $J_{\mathrm{MX}}=7 \mathrm{~Hz}$; (b) $\mathrm{AMX}_{3}$ system with $J_{\mathrm{MX}}=7 \mathrm{~Hz}$; (c) $\mathrm{A}_{3} \mathrm{MQX}_{3}$ system with $J_{\mathrm{AM}}=J_{\mathrm{MX}}=7 \mathrm{~Hz}, J_{\mathrm{AQ}}=J_{\mathrm{XQ}}=0$; (d) $\mathrm{AMQ}_{3} \mathrm{X}_{3}$ system with $J_{\mathrm{MQ}}=J_{\mathrm{MX}}=7 \mathrm{~Hz}, J_{\mathrm{AQ}}=J_{\mathrm{QX}}=0$; (e) AMQX system with $J_{\mathrm{MQ}}=14 \mathrm{~Hz}$ and $J_{\mathrm{MX}}=J_{\mathrm{QX}}=J_{\mathrm{AQ}}=7 \mathrm{~Hz}$; (f) AMQX system with $J_{\mathrm{MQ}}=J_{\mathrm{MX}}=7 \mathrm{~Hz}, J_{\mathrm{AQ}}=0$; (g) $\mathrm{AMPQX}_{3}$ system with $J_{\mathrm{MP}}=J_{\mathrm{MQ}}=J_{\mathrm{MX}}=7$ Hz. Intensity " 1 " corresponds approximately to the intensity of an AX cross peak that would be observed in a hypothetical spin system that differs from the one mentioned only by omission of spin $M$ and by introduction of a coupling $J_{\mathrm{AX}}=\left(\left|J_{\mathrm{AM}}\right|+\left|J_{\mathrm{MX}}\right|\right) / 2$. 
(2) Spin A is coupled to spin $M$, and spin $M$ is coupled to both $Q$ and $X$. This case occurs, for example, for transfer from an amide proton to two nonequivalent $\beta$ protons. The transfer function is then given by

$$
F(\tau)=\sin \left(\pi J_{\mathrm{AM}} \tau\right) \sin \left(\pi J_{\mathrm{MX}} \tau\right) \cos \left(\pi J_{\mathrm{MQ}} \tau\right)
$$

This function is sketched in Fig. $3 \mathrm{f}$.

$A M P Q X_{3}$ spin system. The transfer function for the $\mathrm{AMPQX}_{3}$ spin system is appropriate for transfer from the $\alpha$ proton to the $\gamma$-methyl group in isoleucine. The transfer function is given by

$$
F(\tau)=3 \sin \left(\pi J_{\mathrm{AM}} \tau\right)\left[\sin \left(\pi J_{\mathrm{MX}} \tau\right)+\sin \left(3 \pi J_{\mathrm{MX}} \tau\right)\right]\left[\cos \left(\pi J_{\mathrm{MQ}} \tau\right) \cos \left(\pi J_{\mathrm{MP}} \tau\right)\right] / 4
$$

The shape of this function is sketched in Fig. $3 \mathrm{~g}$.

As mentioned before, the transfer functions [12]-[18] are also valid for cases where $\mathrm{A}$ and $\mathrm{X}$ are coupled to a number of other spins, provided that those other spins have no (or a very small) coupling with spin $M$. The effect of those other spins coupled to $A$ or $X$, is to spread the intensity of the relayed magnetization over a larger number of individual peaks in the relay cross multiplet and the observed maximum intensity for this multiplet will therefore decrease. Exact calculation of the intensity of a cross multiplet in a 2D RELAY spectrum is generally not feasible because it depends strongly on the size of the couplings involved, the $T_{2}$ values, the digital filtering window, and on the lengths of the acquisition times, $t_{1 \max }$ and $t_{2 \max }$. However, an idea about the intensity that is expected for a cross peak in a 2D RELAY spectrum can be obtained from the calculated transfer efficiency. Intensity " 1 " for a cross multiplet between nuclei A and $X$ corresponds approximately to the intensity that would be observed in a COSY spectrum for the cross multiplet between two hypothetical nuclei $\mathrm{A}^{\prime}$ and $\mathrm{X}^{\prime}$ with a coupling constant that is the average of $\left|J_{\mathrm{AM}}\right|$ and $\left|J_{\mathrm{MX}}\right|$, spin $\mathrm{M}$ being absent, but all other passive spins being present. This means that $\mathrm{A}^{\prime}$ and $\mathrm{X}^{\prime}$ would have approximately the same multiplet structure as $A$ and $X$, respectively. For the AMQX system, where two "transfer nuclei," $M$ and $Q$, are present, intensity " 1 " corresponds approximately to the intensity that would be observed in a COSY spectrum for an AQX system where a coupling, $J_{\mathrm{AX}}$ would be present that equals the average of $\left|J_{\mathrm{AM}}\right|$ and $\left|J_{\mathrm{MX}}\right|$, other couplings remaining unchanged compared with the AMQX system.

It is seen from the graphs in Fig. 3 that the intensity of relayed magnetization depends strongly on the size of the coupling $J_{\mathrm{AM}}$; however, the optimum choice for the mixing time is only little affected by variation of the valuc of $J_{\mathrm{AM}}$.

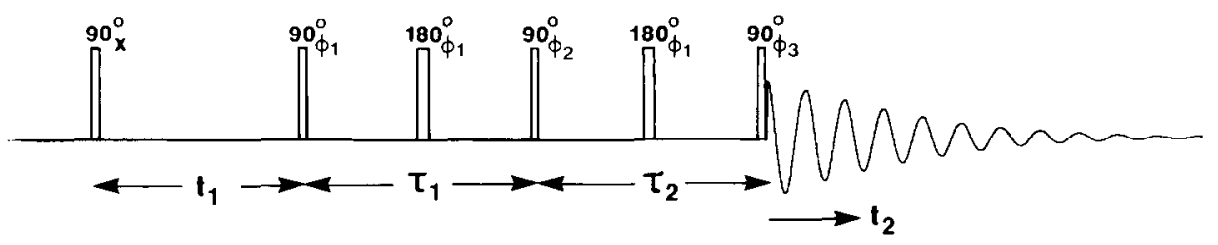

FIG. 4. Pulse scheme of the homonuclear double-RELAY experiment. For optimum results, phases of the various if pulses should be cycled according to Table 2 . 
TABLE 2

The Phases of the rf Pulses and of the Receiver in the Various Steps of the Homonuclear Double-RELAY Experiment, Sketched in Fig. $4^{a}$

\begin{tabular}{rrrrr}
\hline Step & $\phi_{1}$ & $\phi_{2}$ & $\phi_{3}$ & Receiver \\
\hline 1 & $x$ & $x$ & $x$ & + \\
2 & $x$ & $x$ & $-x$ & + \\
3 & $x$ & $-x$ & $x$ & + \\
4 & $x$ & $-x$ & $-x$ & + \\
5 & $y$ & $y$ & $y$ & - \\
6 & $y$ & $y$ & $-y$ & - \\
7 & $y$ & $-y$ & $y$ & - \\
8 & $y$ & $-y$ & $-y$ & + \\
9 & $-x$ & $-x$ & $-x$ & + \\
10 & $-x$ & $-x$ & $x$ & + \\
11 & $-x$ & $x$ & $-x$ & - \\
12 & $-x$ & $-y$ & $x$ & - \\
13 & $-y$ & $-y$ & $-y$ & - \\
14 & $-y$ & $y$ & $y$ & - \\
15 & $-y$ & $y$ & $-y$ & + \\
16 & $-y$ & $x$ & $y$ & + \\
\hline
\end{tabular}

${ }^{a}$ The entire 16-step sequence should be repeated four times, with phases of all pulses and of the receiver incremented by $90^{\circ}$ each time.

\section{MUI.TIPI.F SIGNAI，REI AY}

As mentioned by Eich et al. (1), double-signal RELAY experiments are feasible. Such experiments allow, for example, correlation of the amide proton with the methyl protons in valine. We present the simplest efficient scheme that is suitable for this purpose. The pulse scheme is set out in Fig. 4. A 16-step phase cycle (Table

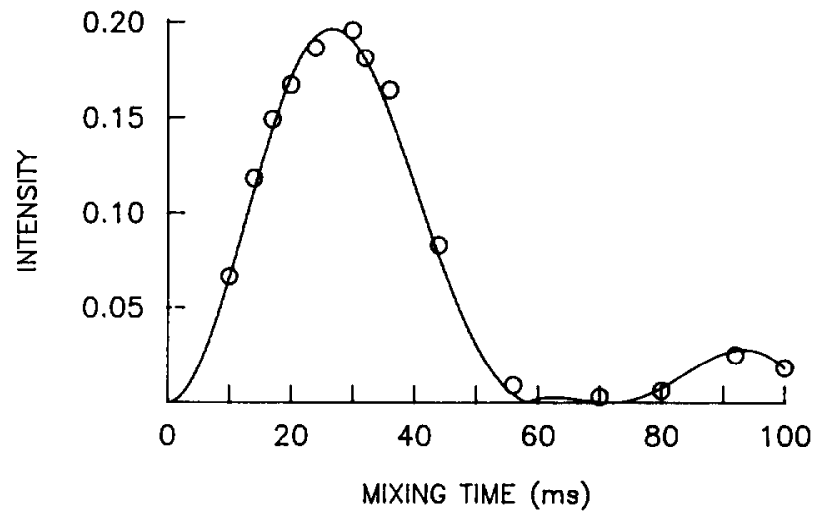

FIG. 5. Integrated intensity of the RELAY cross multiplet between the $\alpha$ proton and $\gamma$-methyl protons in isoleucine, dissolved in ${ }^{2} \mathrm{H}_{2} \mathrm{O}$, at $\mathrm{pH}$ 2.7. Each measured cross peak intensity was obtained from a complete 2D RELAY experiment with full phase cycling (32-step). The drawn line represents the normalized calculated intensity. 


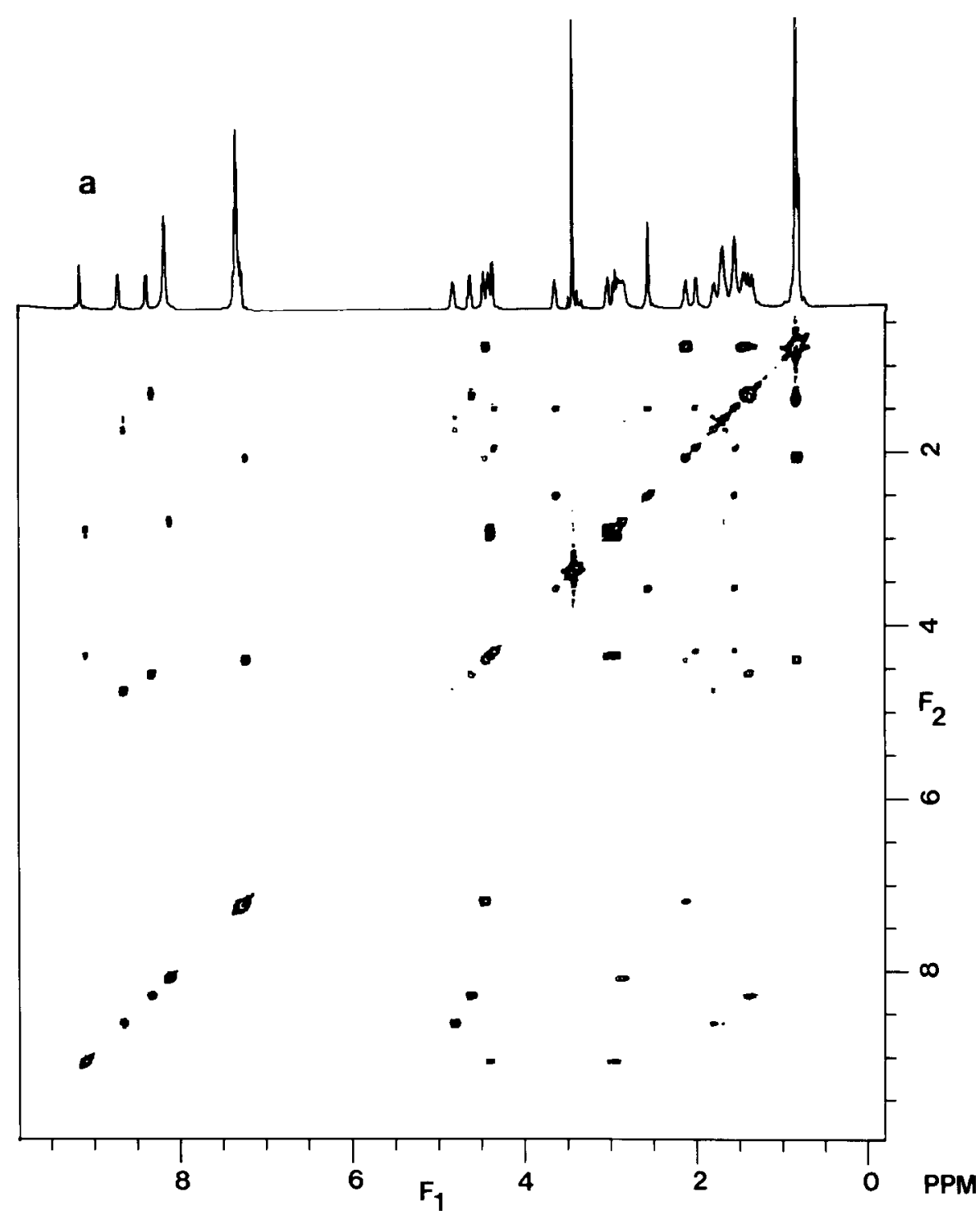

FIG. 6. $500 \mathrm{MHz}$ spectra of gramacidin-S, c(Phe-Leu-Orn-Val-Pro $)_{2}$ in DMSO- $d_{6}$. (a) Two-dimensional RELAY spectrum obtained from a $512 \times 1024$ data matrix, with 32 acquisitions per $t_{1}$ value. The mixing time, $\tau$, was $36 \mathrm{~ms}$. (b) Two-dimensional double-RELAY spectrum, also obtained from a $512 \times 1024$ data matrix, with 64 acquisitions for each $t_{1}$ value. Both halves of the mixing period, $\tau_{1}$ and $\tau_{2}$, were equal to $36 \mathrm{~ms}$. Both spectra are presented in the absolute value mode, and identical data processing has been used for the two spectra. Along the $F_{2}$ axis, a regular ${ }^{1} H$ FID spectrum is shown. Double-RELAY cross peaks between the amide and methyl protons in the Val residue, and the $\alpha$ and methyl protons in the Leu residue are indicated by arrows.

2 ) is needed in this experiment to minimize unwanted COSY and "single-RELAY" correlations. The entire 16-step experiment is then repeated four times in the usual fashion by incrementing the phases of all pulses and of the receiver by $90^{\circ}$ each 


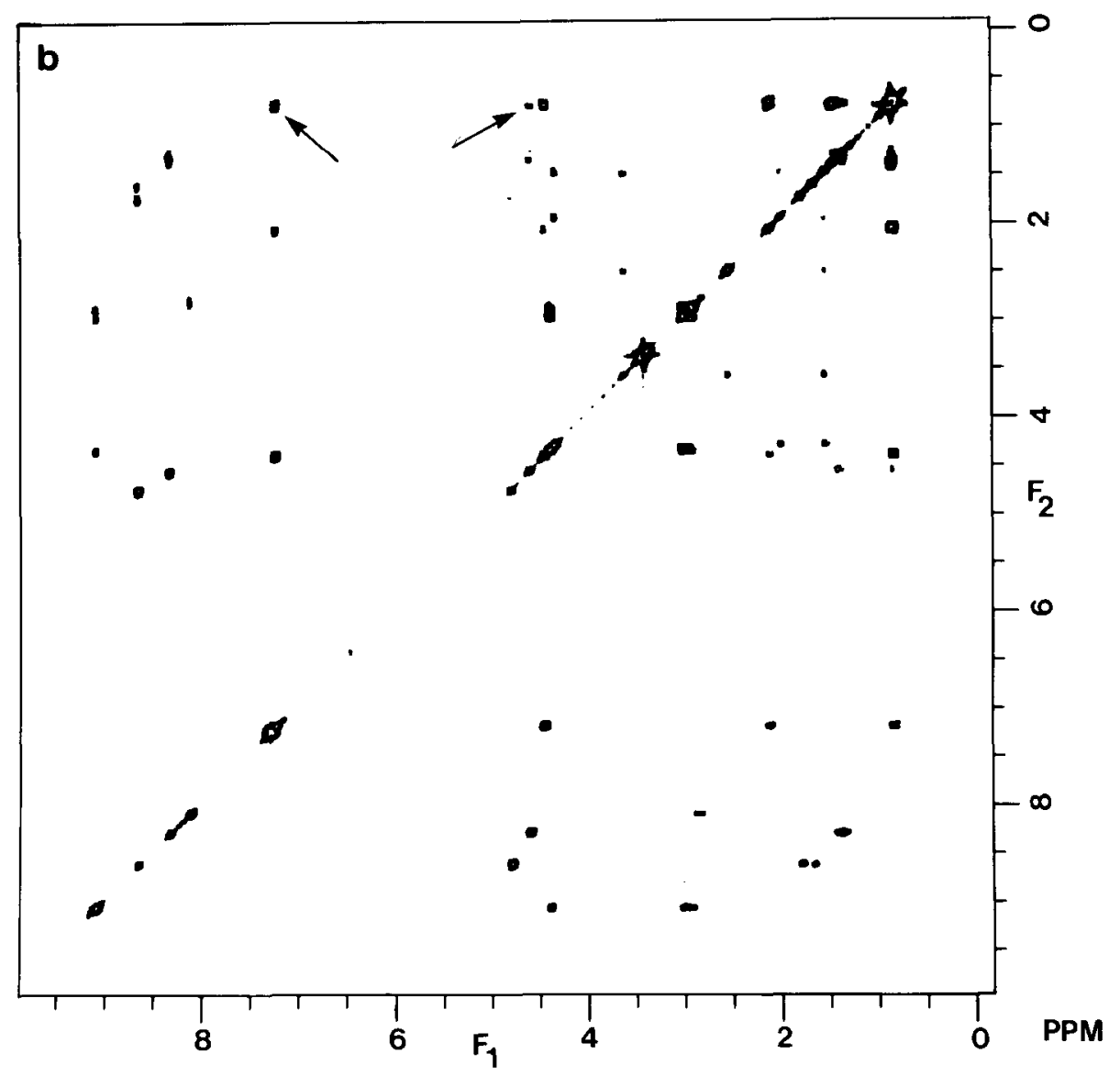

FlG. 6-Continued.

time, in order to eliminate quadrature artifacts (15). The mixing time now consists of two halves, $\tau_{1}$ and $\tau_{2}$, both with a $180^{\circ}$ pulse in the center. For an $\mathrm{A} \rightarrow \mathrm{M} \rightarrow$ $\mathrm{Q} \rightarrow \mathrm{X}$ double-RELAY transfer, during the first half of the mixing period, $\tau_{1}$, magnetization is transferred from $A$ through $M$ to $Q$ (after the second $90^{\circ}$ mixing pulse). During the second half of the mixing period $\left(\tau_{2}\right)$, this Q-spin magnetization is transferred to nucleus $X$. The total transfer efficiency equals the product of the transfer efficiency from $A$ to $Q$ (for mixing time $\tau_{1}$ ) and the transfer efficiency from $\mathrm{M}$ to $\mathrm{X}$ (for mixing time $\tau_{2}$ ). Both factors can be calculated using expressions [11][18]. Note that if $\tau_{1}$ and $\tau_{2}$ have different durations, the two doubly relayed cross peaks will generally differ in intensity.

\section{EXPERIMENTAL VERIFICATION}

A large number of spin systems has been investigated to check the correctness of the expressions presented in the previous section. Experiments have been performed on a Bruker CXP $200 \mathrm{MHz}$ spectrometer, a Nicolet $270 \mathrm{MHz}$ spectrometer and on 

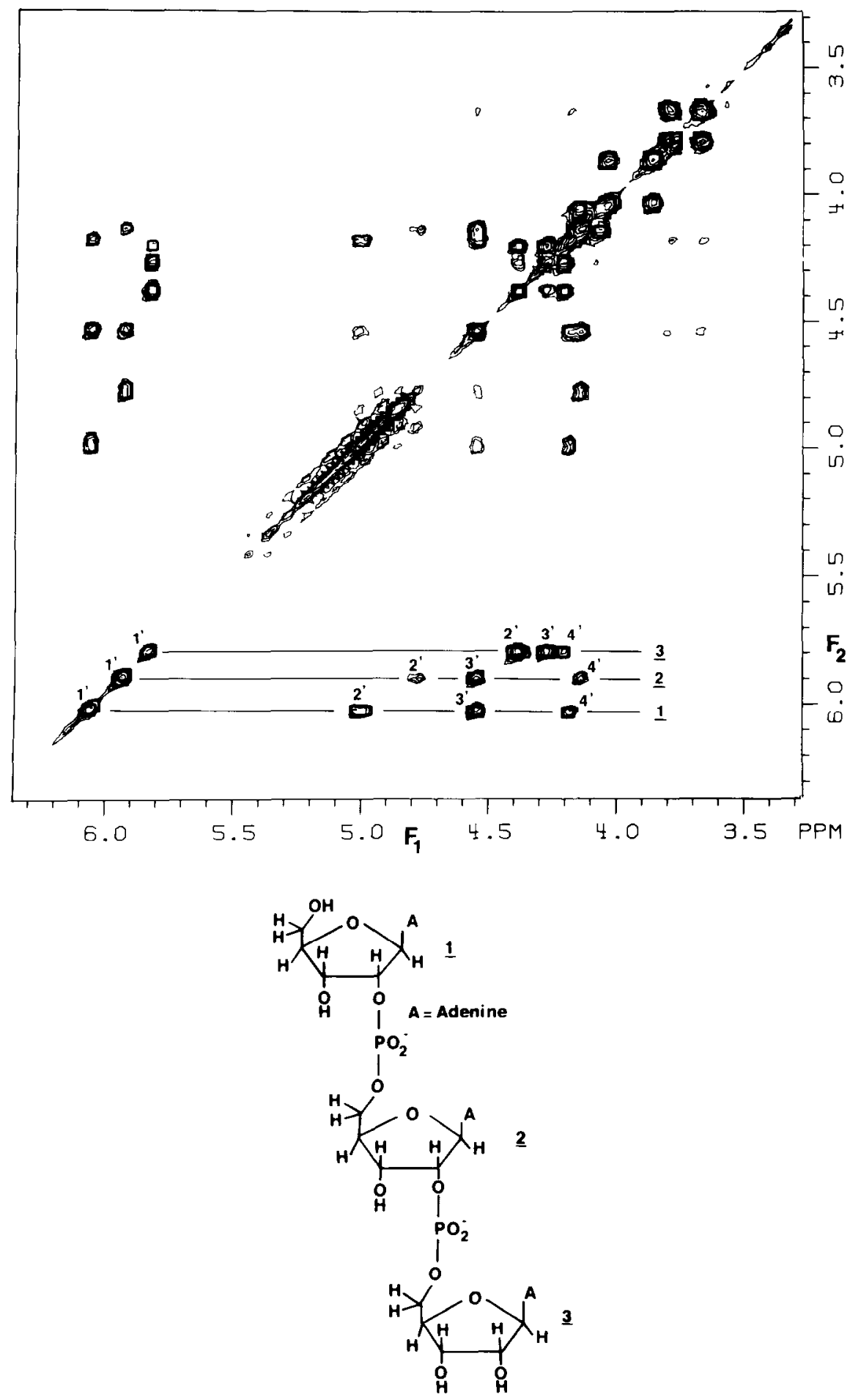

FIG. 7. High-field region of the $500 \mathrm{MHz}$ double-RELAY spectrum of the trinucleotide $\mathrm{A} 2^{\prime}-5^{\prime} \mathrm{A} 2^{\prime}-5^{\prime} \mathrm{A}$. The spectrum is obtained from a $256 \times 512$ data matrix, with 64 acquisitions per $t_{1}$ value. The total measuring time was $9 \mathrm{~h}$. Both halves of the mixing time, $\tau_{1}$ and $\tau_{2}$, were $60 \mathrm{~ms}$. 
a Nicolet $500 \mathrm{MHz}$ spectrometer. All results were qualitatively in good agreement with theory. Of course, the graphs, presented in Fig. 3, give only a qualitative impression of what to expect for a certain spin system. For actual use, one has to substitute the real values of the scalar couplings in expressions [11]-[18]. Here, results will be presented for a sample of isoleucine, dissolved in ${ }^{2} \mathrm{H}_{2} \mathrm{O}$, at $\mathrm{pH} 2.7$. The $\alpha, \beta$, and $\gamma$ protons form an $\mathrm{AMPQX}_{3}$ spin system, with $J_{\mathrm{AM}}=4.0 \mathrm{~Hz}$, $J_{\mathrm{MX}}=7.0 \mathrm{~Hz}, J_{\mathrm{MP}}=4.8 \mathrm{~Hz}$ and $J_{\mathrm{MQ}}=8.7 \mathrm{~Hz}$. Fourteen two-dimensional experiments were performed, and the integrated intensities for the AX RELAY cross peak are shown in Fig. 5, showing good agreement with theoretical predictions.

Figure 6 shows the single- and double-RELAY spectra for a $50 \mathrm{mM}$ solution of gramacidin-S in DMSO- $d_{6}$. The experiments were performed at $500 \mathrm{MHz}$, and full phase cycling (32-step and 64-step, respectively) was used. The single-RELAY spectrum (Fig. 6a) shows connectivity between all four amide protons and the corresponding $\beta$ protons. The double-RELAY spectrum (Fig. 6b) shows a number of COSY and single-RELAY peaks, and also two double-RELAY peaks, indicated by arrows. For example, the amide/methyl cross peaks for the valine residue have rather high intensity and immediately identify those resonances. Intense amide/ methyl cross peaks for valine residues have also been observed in a small protein, currently under investigation.

Figure 7 shows the double-RELAY spectrum for the trinucleotide $A 2^{\prime}-5^{\prime} \mathrm{A} 2^{\prime}-5^{\prime} \mathrm{A}$, obtained at $500 \mathrm{MHz}$. The spectrum shows that protons $1^{\prime}$ to $4^{\prime}$ can all be correlated with relatively high intensity, giving an unambiguous subspectrum for each individual sugar unit by taking a cross section parallel to the $F_{1}$ or $F_{2}$ axis at the $F_{2}$ or $F_{1}$ frequency of the corresponding anomeric proton.

\section{DISCUSSION}

It is clear from the analysis of the experiment, that optimum signal relay depends not only on the size of the coupling constants involved, but also on the type of spin system. At first sight it may appear disappointing that no single optimum value for the mixing time in the RELAY experiment exists. However, the fact that different spin systems show optimum RELAY cross peaks for different durations of the mixing time can also be advantageous; by performing a number of RELAY experiments with different durations of the mixing time one may be able to identify certain types of spin systems directly by analyzing the RELAY cross peak intensity as a function of the mixing time. Since one often wants to observe peaks with very low intensity, it is important to keep the spectrum as free as possible from artefacts and intense resonances that are of no interest (diagonal and COSY-type peaks). The 32-step phase cycling described earlier is optimal for this purpose. Further phase cycling does not significantly improve the spectrum and merely increases the minimum measuring time for the experiment. With the phase cycling described in this paper we find it unnecessary to place the transmitter at the low or high-field side of the spectrum and thus a minimum size of the data matrix can be employed. Possible nonsuppressed $p$-type and axial peaks (8) could not be detected, even though in some of the experiments the signal-to-noise ratio on traces parallel to the $F_{1}$ axis was higher than $800: 1$. A relaxation delay equal to $1.5 T_{1}$ between successive scans is close to optimum from a sensitivity point of view, and is sufficiently long 
to avoid the introduction of artifacts. The use of a non-shifted sine-bell filter is close to optimum since (a) it matches approximately the envelope amplitude of the signal components that contribute to a RELAY cross peak, (b) it partially suppresses unwanted diagonal and COSY-type peaks, and (c) it improves resolution in the absolute-value mode.

Double-RELAY experiments are also feasible. The intensities of double- and multiple-RELAY cross peaks can be predicted in a similar way as described above. This experiment is particularly useful if no nonequivalent geminal protons are present, due to the relatively large amount of transfer that can be obtained in this case. We have found the experiment very powerful in the study of complex carbohydrates and polynucleotides, as well as for the assignment of resonances in large peptides and small proteins.

\section{ACKNOWLEDGMENTS}

Gary Drobny acknowledges financial support from the Murdock Charitable Trust and from NIH Grant 1 PO1 GM32681-01. Ad Bax is indebted to Rolf Tschudin for continuous technical support and to Dr. Paul Torrens for providing a sample of the trinucleotide A2'-5'A2'-5'A.

Note added in proof. Results very similar to those presented in this paper were derived independently by $\mathbf{H}$. Widmer, G. Wagner and K. Wüthrich and presented at the XXII Congress Ampere on Magnetic Resonance and Related Phenomena, September 1984, Zürich, Switzerland.

\section{REFERENCES}

I. G. Eich, G. BodenhauSEn, AND R. R. ERNST, J. Am. Chem. Soc. 104, 3732 (1982).

2. J. Jeener, Ampere International Summer School, Basko Polje, Yugoslavia, 1971.

3. W. P. Aue, E. Bartholdi, and R. R. ERnst, J. Chem. Phys. 64, 2229 (1976).

4. A. BaX, R. Freeman, AND G. A. Morris, J. Magn. Reson. 42, 164 (1982).

5. K. Nagayama, A. Kumar, K. WÜthrich, and R. R. ERnSt, J. Magn. Reson. 40, 321 (1980).

6. A. BaX AND R. Freeman, J. Magn. Reson. 44, 542 (1981).

7. A. Bax, "Two-Dimensional Nuclear Magnetic Resonance in Liquids," Chap. 2, Reidel, Boston, 1982.

8. G. Wider, S. Macura, A. Kumar, R. R. ERnSt, AND K. WÜthrich, J. Magn. Reson. 56, 207 (1984).

9. U. Piantini, O. W. Sørensen, and R. R. ERnst, J. Am. Chem. Soc. 104, 6800 (1982).

10. K. Nagayama, K. Wüthrich, AND R. R. ERnst, Biochem. Biophys. Res. Commun. 90, 305 (1979).

11. G. WAGNER, J. Magn. Reson. 55, 151 (1983).

12. G. KING AND P. WRIGHT, J. Magn. Reson. 54, 328 (1983).

13. O. W. Sørensen, G. W. Eich, M. H. LeVitt, G. Bodenhausen, and R. R. ERnst, Progr. Nucl. Magn. Reson. Spectrosc. 16, 163 (1983).

14. D. I. Hoult and R. E. Richards, Proc. Roy. Soc. London Ser. A 344, 311 (1975).

15. G. Bodenhausen, R. Freeman, AND D. L. TuRner, J. Magn. Reson. 27, 511 (1977).

16. G. Bodenhausen, H. Kogler, AND R. R. ERnSt, J. Magn. Keson. 58, 370 (1984).

17. R. R. ERNST, in "Advances in Magnetic Resonance" (J. S. Waugh, Ed.), Vol. 2, pp. 1-135, Academic Press, New York, 1966.

18. A. De Marco AND K. WUUthrich, J. Magn. Reson. 24, 201 (1976).

19. I. D. Campbell, C. M. Dobson, R. J. P. Williams, and A. V. Xavier, J. Magn. Reson. 11, 172 (1973).

20. A. BAX, R. FreEMAN, AND G. A. MORRIS, J. Magn. Reson. 43, 333 (1981). 